\title{
INCIDENCE RELATION FOR PRIMES OF A GLOBAL FUNCTION FIELD
}

\author{
ALFRED CZOGAŁA and PRZEMYSŁAW KOPROWSKI \\ Institute of Mathematics, University of Silesia \\ Bankowa 14, 40-007 Katowice, Poland \\ ORCID: 0000-0002-3118-2404, 0000-0003-0952-5738 \\ E-mail: alfred.czogala@us.edu.pl,przemyslaw.koprowski@us.edu.pl
}

\begin{abstract}
We show that primes of any global function field (of characteristic $\neq 2$ and containing a square root of -1 ) form a connected graph of diameter 2 . This fact generalizes our earlier result concerning the graph of even points.
\end{abstract}

Let us begin with an elementary example that motivates our further considerations. Take two irreducible polynomials $f, g \in \mathbb{F}_{q}[t]$ with coordinates in a finite field of odd characteristic. The quadratic reciprocity law asserts that

$$
\left(\frac{f}{g}\right)\left(\frac{g}{f}\right)=(-1)^{(q-1)(\operatorname{deg} f \operatorname{deg} g) / 2} .
$$

This leads to a definition of a relation on the set of irreducible polynomials. Write $f \smile g$ if $\left(\frac{f}{g}\right)=1$. By the quadratic reciprocity law this relation is symmetric unless $q \equiv 3(\bmod 4)$ and $f, g$ have odd degrees. In this case, $\smile$ is antisymmetric. Anyway, in general this relation is not transitive. Take for instance $\mathbb{F}_{q}=\mathbb{F}_{11}$ and three polynomials

$$
f=t^{3}+t^{2}+2, \quad g=t^{3}+t^{2}+3 t+1, \quad h=t^{3}+2 t+2 .
$$

Then $f \smile g$ and $g \smile h$ but $f \nsucc h$. Thus it is natural to ask about the transitive closure of $\smile$.

Assume that $q \equiv 1(\bmod 4)$ so the relation is symmetric. One can show that for every two polynomials $f, g \in \mathbb{F}_{q}[t]$, if $f \nsucc g$, then there is a polynomial $h \in \mathbb{F}_{q}[t]$ such that

2010 Mathematics Subject Classification: 11G20, 11R65, 11R45, 14C22, 05C40.

Key words and phrases: global function fields, curves over finite fields, Picard groups, connected graphs, graph's diameter.

The paper is in final form and no version of it will be published elsewhere. 
$f \smile h$ and $h \smile g$. In other words, the set of irreducible polynomials with relation $\smile$ forms a connected graph of diameter 2 .

Let $K=\mathbb{F}_{q}(X)$ be an arbitrary global function field of characteristic $\neq 2$. A generalization of the relation $\smile$ to the set of points (primes) of $K$, whose classes are 2-divisible in the Picard group Pic $X$, was introduced in [2] and further investigated in [3, 1]. We should emphasize the fact that on such points the relation $\smile$ is defined canonically. In [1] we show that for every global function field (of odd characteristic) the corresponding graph is connected and has diameter 2. The purpose of the present note is to show that one may generalize the relation $\smile$ to all points of $K$, not only those whose classes in Pic $X$ are 2-divisible. Thus this paper may be viewed as an addendum to [1. Unfortunately the generalized relation is no longer canonical. It depends on a choice of a certain system of parameters $\pi_{1}, \ldots, \pi_{k}$, where $k$ is the dimension of Pic $X / 2$ Pic $X$, treated as a $\mathbb{F}_{2}$-vector space. In case of the field $K=\mathbb{F}_{q}(t)$ of rational functions, this choice corresponds to the selection of the 'variable': an element transcendental over the ground field and generating $K$.

Throughout this paper $K$ denotes a global function field of odd characteristic and $\mathbb{F}_{q}$ is its full field of constants. Further, $X$ is the set of primes (equivalent classes of valuations) on $K$. From a geometric point of view, $X$ is a smooth complete algebraic curve over $\mathbb{F}_{q}$. If $Y$ is a nonempty Zariski-open subset of $X$, then $Y$ is an affine curve and by $\operatorname{Pic} Y$ we denote the Picard group of $Y$, i.e. the quotient of the group $\operatorname{Div} Y$ of $Y$-divisors modulo the principal divisors. Given a point $\mathfrak{p} \in X$, by $[\mathfrak{p}]$ we denote its class in Pic $X$, by $K_{\mathfrak{p}}$ the completion of $K$ with respect to $\mathfrak{p}$ and by $\operatorname{ord}_{\mathfrak{p}}$ the associated discrete valuation $K \rightarrow \mathbb{Z} \cup\{\infty\}$. Moreover, with every open subset $Y \subseteq X$ we associate two subgroups of the square-class group of $K$ :

$$
\begin{aligned}
& \mathbf{E}_{Y}:=\left\{\lambda \in K^{\times} / K^{\times 2} \mid \operatorname{ord}_{\mathfrak{p}} \lambda \equiv 0(\bmod 2) \text { for every } \mathfrak{p} \in Y\right\}, \\
& \boldsymbol{\Delta}_{Y}:=\left\{\lambda \in \mathbf{E}_{Y} \mid \lambda \in K_{\mathfrak{p}}^{\times 2} \text { for every } \mathfrak{p} \in X \backslash Y\right\} .
\end{aligned}
$$

Definition. A finite set $\left\{\pi_{1}, \ldots, \pi_{k}\right\} \subset K$ will be called a system of parameters for $\smile$ if there are points $\mathfrak{b}_{1}, \ldots, \mathfrak{b}_{k} \in X$ such that:

- $\operatorname{ord}_{\mathfrak{b}_{i}} \pi_{i}=1$ for every $i \leq k$,

- $\left\{\left[\mathfrak{b}_{1}\right]+2 \operatorname{Pic} X, \ldots,\left[\mathfrak{b}_{k}\right]+2 \operatorname{Pic} X\right\}$ is a basis of $\operatorname{Pic} X / 2 \operatorname{Pic} X$.

We need the following three facts from [1]:

Proposition 1 ([1, Lemma 2]). Let $\left\{\pi_{1}, \ldots, \pi_{k}\right\}$ be a system of parameters for $\smile$ and $\mathfrak{B}=\left\{\mathfrak{b}_{1}, \ldots, \mathfrak{b}_{k}\right\}$ a corresponding set of points of $X$. Then there is a basis $\mathscr{B}=$ $\left\{\beta_{1}, \ldots, \beta_{k}\right\}$ of $\mathbf{E}_{X}$ such that

$$
\left(\frac{\beta_{i}}{\mathfrak{b}_{j}}\right)= \begin{cases}1 & \text { if } i \neq j \\ -1 & \text { if } i=j\end{cases}
$$

for all $i, j \leq k$. 
Proposition 2 ([1, Proposition 7]). Let $\mathfrak{b}_{1}, \ldots, \mathfrak{b}_{k}$ and $\beta_{1}, \ldots, \beta_{k}$ be as in the previous proposition. Further let $\mathfrak{p} \in X$, then

$$
[\mathfrak{p}] \equiv \sum_{i \leq k} \varepsilon_{i} \cdot\left[\mathfrak{b}_{i}\right](\bmod 2 \operatorname{Pic} \mathrm{X})
$$

if and only if

$$
\left(\frac{\beta_{i}}{\mathfrak{p}}\right)=(-1)^{\varepsilon_{i}} \quad \text { for every } i \leq k .
$$

Proposition 3 ([1, Proposition 6$])$. If $\mathfrak{p}, \mathfrak{q} \in X$ are two points whose classes are congruent modulo 2 Pic $X$, then $\boldsymbol{\Delta}_{X \backslash\{\mathfrak{p}\}}=\boldsymbol{\Delta}_{X \backslash\{\mathfrak{q}\}}$.

For the rest of this paper we will work under the following assumptions:

(AS1) $-1 \in K^{\times 2}$

(AS2) $\Pi=\left\{\pi_{1}, \ldots, \pi_{k}\right\} \subset K$ is a fixed system of parameters for $\smile$,

(AS3) $\mathfrak{B}=\left\{\mathfrak{b}_{1}, \ldots, \mathfrak{b}_{k}\right\} \subset X$ is the corresponding set of points such that ord $_{\mathfrak{b}_{i}} \pi_{i}=1$ and $\left\{\left[\mathfrak{b}_{i}\right]+2 \operatorname{Pic} X \mid i \leq k\right\}$ is a basis of $\operatorname{Pic} X / 2 \operatorname{Pic} X$,

(AS4) $\mathscr{B}=\left\{\beta_{1}, \ldots, \beta_{k}\right\}$ is a basis of $\mathbf{E}_{X}$ such that $\left(\frac{\beta_{i}}{\mathfrak{b}_{j}}\right)=-1$ if and only if $i=j$.

In addition we denote the affine curve $X \backslash \mathfrak{B}$ by $Z$.

Remark. Take a finite field $\mathbb{F}_{q}$ such that $-1 \in \mathbb{F}_{q}^{\times 2}$ and let $\zeta \in \mathbb{F}_{q}$ be a fixed non-square. Consider the field $K=\mathbb{F}_{q}(t)$ of rational functions and take $\pi:=1 / t$. Finally, let $\mathfrak{b}$ be the "point at infinity", i.e. $\mathfrak{b}=\{f / g \in K \mid \operatorname{deg} g>\operatorname{deg} f\}$. Then $\Pi=\{\pi\}, \mathfrak{B}=\{\mathfrak{b}\}$ and $\mathscr{B}=\{\zeta\}$ satisfy conditions AS1 AS4

Proposition 4. For every $\mathfrak{p} \in Z$ one has

(1) $\mathbf{E}_{Z}=\mathbf{E}_{X}$

(2) $\left[\mathbf{E}_{Z \backslash\{\mathfrak{p}\}}: \mathbf{E}_{X}\right]=2$.

Proof. The first assertion follows immediately from [3, Lemma 2.3]. We shall prove the other one. By [2, Proposition 2.3] we have

$$
\begin{aligned}
\mathrm{rk}_{2} \mathbf{E}_{Z} & =\operatorname{rk}_{2} \operatorname{Pic} Z+|\mathfrak{B}|, \\
\operatorname{rk}_{2} \mathbf{E}_{Z \backslash\{\mathfrak{p}\}} & =\operatorname{rk}_{2} \operatorname{Pic}(Z \backslash\{\mathfrak{p}\})+|\mathfrak{B}|+1 .
\end{aligned}
$$

On the other hand, [3, Proposition 2.4] yields

$$
\operatorname{rk}_{2} \operatorname{Pic} Z=\operatorname{rk}_{2} \operatorname{Pic}^{0} X-|\mathfrak{B}|+1=\operatorname{rk}_{2} \operatorname{Pic}(Z \backslash\{\mathfrak{p}\}) .
$$

Combining Eq. (1) with Eq. (2) we obtain

$$
\mathrm{rk}_{2} \mathbf{E}_{Z \backslash\{\mathfrak{p}\}}=\operatorname{rk}_{2} \operatorname{Pic}^{0} X+2=\operatorname{rk}_{2} \mathbf{E}_{Z}+1
$$

and this concludes the proof.

Proposition 5. Let $\mathfrak{p} \in X$ be a point.

(1) If $[\mathfrak{p}] \in 2 \operatorname{Pic} X$, then $\left[\mathbf{E}_{Z \backslash\{\mathfrak{p}\}}: \boldsymbol{\Delta}_{X \backslash\{\mathfrak{p}\}}\right]=2$.

(2) If $[\mathfrak{p}] \notin 2 \operatorname{Pic} X$, then $\left[\mathbf{E}_{Z \backslash\{\mathfrak{p}\}}: \boldsymbol{\Delta}_{X \backslash\{\mathfrak{p}\}}\right]=4$. 
Proof. By [2, Proposition 3.4] the class of $\mathfrak{p}$ is 2-divisible in Pic $X$ if and only if $\mathbf{E}_{X}=$ $\boldsymbol{\Delta}_{X \backslash\{\mathfrak{p}\}}$. Hence the first assertion follows immediately from the preceding proposition. Now assume that $[\mathfrak{p}] \notin 2$ Pic $X$. Therefore

$$
\operatorname{rk}_{2} \Delta_{X \backslash\{\mathfrak{p}\}}=\operatorname{rk}_{2} \operatorname{Pic}(X \backslash\{\mathfrak{p}\})
$$

by [2, Proposition 2.3] and

$$
\operatorname{rk}_{2} \operatorname{Pic}(X \backslash\{\mathfrak{p}\})=\operatorname{rk}_{2} \operatorname{Pic}^{0} X
$$

by [2, Proposition 2.7]. Finally [2, Lemma 2.4] asserts that $\mathrm{rk}_{2} \mathbf{E}_{X}=1+\mathrm{rk}_{2} \mathrm{Pic}^{0} X$. Consequently we have $\left[\mathbf{E}_{X}: \boldsymbol{\Delta}_{X \backslash\{\mathfrak{p}\}}\right]=2$ and so Proposition 4 implies the second assertion, as well.

Assume that $\mathfrak{p} \in X$ and $[\mathfrak{p}] \notin 2 \operatorname{Pic} X$. The group $\mathbf{E}_{Z \backslash\{\mathfrak{p}\}} / \boldsymbol{\Delta}_{X \backslash\{\mathfrak{p}\}}$ is isomorphic to the Klein 4-group since $\mathbf{E}_{Z \backslash\{\mathfrak{p}\}}$ and $\boldsymbol{\Delta}_{X \backslash\{\mathfrak{p}\}}$ are both elementary 2-groups. We are going to explicitly describe the four cosets of $\boldsymbol{\Delta}_{X \backslash\{\mathfrak{p}\}}$.

Lemma 6. Let $\mathfrak{q} \in Z$ be a point (not necessarily distinct from $\mathfrak{p}$ ). Assume that the classes of $\mathfrak{p}$ and $\mathfrak{q}$ are congruent modulo 2 Pic $X$. Then the cosets of $\boldsymbol{\Delta}_{X \backslash\{\mathfrak{p}\}}$ are contained in square classes of $K_{\mathfrak{q}}$.

Proof. Assume that $\lambda, \mu \in \mathbf{E}_{Z \backslash\{\mathfrak{p}\}}$ and $\lambda \equiv \mu\left(\bmod \boldsymbol{\Delta}_{\mathrm{X} \backslash\{\mathfrak{p}\}}\right)$. It follows from Proposition 3 that $\lambda \mu \in \boldsymbol{\Delta}_{X \backslash\{\mathfrak{p}\}}=\boldsymbol{\Delta}_{X \backslash\{\mathfrak{q}\}} \subset K_{\mathfrak{q}}^{\times 2}$.

Lemma 7. Let $\mathfrak{p} \in Z$ be a point. Assume that

$$
[\mathfrak{p}] \equiv \sum_{i \leq k} \varepsilon_{i} \cdot\left[\mathfrak{b}_{i}\right](\bmod 2 \operatorname{Pic} \mathrm{X})
$$

for some $\varepsilon_{1}, \ldots, \varepsilon_{k} \in \mathbb{F}_{2}$. Then there is a square class $\lambda_{\mathfrak{p}} \in \mathbf{E}_{Z \backslash\{\mathfrak{p}\}}$ such that

(1) $\operatorname{ord}_{\mathfrak{p}} \lambda_{\mathfrak{p}} \equiv 1(\bmod 2)$,

(2) $\lambda_{\mathfrak{p}} \in K_{\mathfrak{b}_{i}}^{\times 2}$ for $i \leq k$ such that $\varepsilon_{i}=0$,

(3) $\lambda_{\mathfrak{p}} \in \pi_{i} K_{\mathfrak{b}_{i}}^{\times 2}$ for $i \leq k$ such that $\varepsilon_{i}=1$.

Proof. By the assumption there is $\mu \in K^{\times}$and $\mathcal{D} \in \operatorname{Div} X$ such that

$$
\operatorname{div}_{X} \mu=\mathfrak{p}+\sum_{i \leq k} \varepsilon_{i} \mathfrak{b}_{i}+2 \mathcal{D} .
$$

Define

$$
A:=\left\{i \leq k \mid \mu \notin K_{\mathfrak{b}_{i}}^{\times 2} \cup \pi_{i} K_{\mathfrak{b}_{i}}^{\times 2}\right\}
$$

and set

$$
\lambda_{\mathfrak{p}}:=\mu \cdot \prod_{i \in A} \beta_{i}
$$

It is clear that $\lambda_{\mathfrak{p}}$ satisfies conditions $(1)-(3)$.

REMARK. Let $K=\mathbb{F}_{q}(t)$ be the field of rational functions and $\pi, \mathfrak{b}, \zeta$ be defined in the same way as in the previous remark. If $\mathfrak{p} \in Z$ is generated (as an ideal) by some monic irreducible polynomial $f$, then $\lambda_{\mathfrak{p}}=f$. 
Proposition 8. Let $\mathfrak{p} \in Z$ be a point such that $[\mathfrak{p}] \notin 2$ Pic $X$. Assume that

$$
[\mathfrak{p}] \equiv \sum_{i \leq k} \varepsilon_{i}\left[\mathfrak{b}_{i}\right](\bmod 2 \operatorname{Pic} \mathrm{X})
$$

for some $\varepsilon_{1}, \ldots, \varepsilon_{k} \in \mathbb{F}_{2}$. Further let $j$ be the least index of a nonzero coordinate $\varepsilon_{j}$. Set $\mu_{\mathfrak{p}}:=\beta_{j}$ and let $\lambda_{\mathfrak{p}}$ be defined as in the preceding lemma. Then the group $\mathbf{E}_{Z \backslash\{\mathfrak{p}\}} / \boldsymbol{\Delta}_{X \backslash\{\mathfrak{p}\}}$ consists of the following four cosets of $\boldsymbol{\Delta}_{X \backslash\{\mathfrak{p}\}}$ :

$$
\boldsymbol{\Delta}_{X \backslash\{\mathfrak{p}\}}, \quad \mu_{\mathfrak{p}} \cdot \boldsymbol{\Delta}_{X \backslash\{\mathfrak{p}\}}, \quad \lambda_{\mathfrak{p}} \cdot \boldsymbol{\Delta}_{X \backslash\{\mathfrak{p}\}} \quad \text { and } \quad \mu_{\mathfrak{p}} \lambda_{\mathfrak{p}} \cdot \boldsymbol{\Delta}_{X \backslash\{\mathfrak{p}\}} \cdot
$$

Proof. By Proposition 2 we have

$$
\left(\frac{\mu_{\mathfrak{p}}}{\mathfrak{p}}\right)=\left(\frac{\beta_{j}}{\mathfrak{p}}\right)=(-1)^{\varepsilon_{j}}=-1,
$$

hence $\mu_{\mathfrak{p}} \notin K_{\mathfrak{p}}^{\times 2}$. Consequently $\boldsymbol{\Delta}_{X \backslash\{\mathfrak{p}\}} \neq \mu_{\mathfrak{p}} \cdot \boldsymbol{\Delta}_{X \backslash\{\mathfrak{p}\}}$ and $\lambda_{\mathfrak{p}} \cdot \boldsymbol{\Delta}_{X \backslash\{\mathfrak{p}\}} \neq \mu_{\mathfrak{p}} \lambda_{\mathfrak{p}} \cdot \boldsymbol{\Delta}_{X \backslash\{\mathfrak{p}\}}$. Further we have $\operatorname{ord}_{\mathfrak{p}} \lambda_{\mathfrak{p}} \equiv 1(\bmod 2)$ and $\operatorname{ord}_{\mathfrak{p}} \mu_{\mathfrak{p}} \equiv 0(\bmod 2)$. It follows that the cosets $\lambda_{\mathfrak{p}} \cdot \boldsymbol{\Delta}_{X \backslash\{\mathfrak{p}\}}, \mu_{\mathfrak{p}} \lambda_{\mathfrak{p}} \cdot \boldsymbol{\Delta}_{X \backslash\{\mathfrak{p}\}}$ differ from $\boldsymbol{\Delta}_{X \backslash\{\mathfrak{p}\}}$ and $\mu_{\mathfrak{p}} \cdot \boldsymbol{\Delta}_{X \backslash\{\mathfrak{p}\}} \cdot$

Recall (see [1]) that points $\mathfrak{p}, \mathfrak{q} \in X$, whose classes are 2-divisible in Pic $X$, are related (denoted $\mathfrak{p} \smile \mathfrak{q})$ if $\mathbf{E}_{X \backslash\{\mathfrak{p}\}} \subset K_{\mathfrak{q}}^{\times 2}$. By [1, Proposition 13] this happens if and only if $\lambda_{\mathfrak{p}} \in K_{\mathfrak{q}}^{\times 2}$. We will use the last property to define the relation $\smile$ on the whole $Z$.

Definition. Let $\mathfrak{p}, \mathfrak{q} \in Z$ be two points, we write $\mathfrak{p} \smile \mathfrak{q}$ when $\lambda_{\mathfrak{p}} \in K_{\mathfrak{q}}^{\times 2}$.

Remark. Again let $K=\mathbb{F}_{q}(t)$ and $\pi, \mathfrak{b}, \zeta$ be defined in the same way as in the previous remarks. Take $\mathfrak{p}, \mathfrak{q} \in Z$ and assume that $\mathfrak{p}$ is generated by a polynomial $f$ and $\mathfrak{q}$ by $g$, where $f, g \in \mathbb{F}_{q}[t]$ are monic and irreducible. Then $\mathfrak{p} \smile \mathfrak{q}$ if and only if $\lambda_{\mathfrak{p}}=f$ is either a quadratic residue (when at least one of the degrees $\operatorname{deg} f, \operatorname{deg} g$ is even) or a non-residue (when both degrees are odd) modulo $g$. This shows that our definition of $\smile$ generalizes the one discussed in the introduction.

Proposition 9. The relation $\smile$ is symmetric.

Proof. Take two points $\mathfrak{p}, \mathfrak{q} \in Z$ and assume that $\mathfrak{p} \smile \mathfrak{q}$, hence $\lambda_{\mathfrak{p}} \in K_{\mathfrak{q}}^{\times 2}$ and consequently the Hilbert symbol $\left(\lambda_{\mathfrak{p}}, \lambda_{\mathfrak{q}}\right)_{\mathfrak{q}}$ vanishes. We are going to prove that $\lambda_{\mathfrak{q}}$ is a local square at $\mathfrak{p}$. To this end we will show that $\left(\lambda_{\mathfrak{p}}, \lambda_{\mathfrak{q}}\right)_{\mathfrak{p}}=1$. Take a point $\mathfrak{r} \in Z$ distinct from $\mathfrak{p}$ and $\mathfrak{q}$. Then $\operatorname{ord}_{\mathfrak{r}} \lambda_{\mathfrak{p}} \equiv \operatorname{ord}_{\mathfrak{r}} \lambda_{\mathfrak{q}} \equiv 0(\bmod 2)$ and so we have

$$
\left(\lambda_{\mathfrak{p}}, \lambda_{\mathfrak{q}}\right)_{\mathfrak{r}}=1 \quad \text { for every } \mathfrak{r} \in Z \backslash\{\mathfrak{p}, \mathfrak{q}\} .
$$

Now take any $\mathfrak{b}_{i} \in \mathfrak{B}$. Let

$$
[\mathfrak{p}] \equiv \sum_{i \leq k} \varepsilon_{i}\left[\mathfrak{b}_{i}\right] \quad \text { and } \quad[\mathfrak{q}] \equiv \sum_{i \leq k} \epsilon_{i}\left[\mathfrak{b}_{i}\right] \quad(\bmod 2 \operatorname{Pic} \mathrm{X})
$$

Now Lemma 7 yields

$$
\left(\lambda_{\mathfrak{p}}, \lambda_{\mathfrak{q}}\right)_{\mathfrak{b}_{i}}= \begin{cases}(1,1)_{\mathfrak{b}_{i}} & \text { if } \varepsilon_{i}=\epsilon_{i}=0 \\ \left(1, \pi_{i}\right)_{\mathfrak{b}_{i}} & \text { if } \varepsilon_{i}=0 \text { and } \epsilon_{i}=1 \\ \left(\pi_{i}, 1\right)_{\mathfrak{b}_{i}} & \text { if } \varepsilon_{i}=1 \text { and } \epsilon_{i}=0 \\ \left(\pi_{i}, \pi_{i}\right)_{\mathfrak{b}_{i}} & \text { if } \varepsilon_{i}=\epsilon_{i}=1\end{cases}
$$

In all four cases the Hilbert symbol $\left(\lambda_{\mathfrak{p}}, \lambda_{\mathfrak{q}}\right)_{\mathfrak{b}_{i}}$ vanishes. 
Using the Hilbert reciprocity law we compute

$$
1=\prod_{\mathfrak{r} \in X}\left(\lambda_{\mathfrak{p}}, \lambda_{\mathfrak{q}}\right)_{\mathfrak{r}}=\prod_{\substack{\mathfrak{r} \in Z \\ \mathfrak{r} \neq \mathfrak{p}, \mathfrak{q}}}\left(\lambda_{\mathfrak{p}}, \lambda_{\mathfrak{q}}\right)_{\mathfrak{r}} \cdot \prod_{i \leq k}\left(\lambda_{\mathfrak{p}}, \lambda_{\mathfrak{q}}\right)_{\mathfrak{b}_{i}} \cdot\left(\lambda_{\mathfrak{p}}, \lambda_{\mathfrak{q}}\right)_{\mathfrak{q}} \cdot\left(\lambda_{\mathfrak{p}}, \lambda_{\mathfrak{q}}\right)_{\mathfrak{p}} .
$$

It follows from the preceding discussion that all the above factors - except possibly the last one-vanish. But then also $\left(\lambda_{\mathfrak{p}}, \lambda_{\mathfrak{q}}\right)_{\mathfrak{p}}=1$. Now $\lambda_{\mathfrak{p}}$ has an odd valuation so the only way the Hilbert symbol can vanish is $\lambda_{\mathfrak{q}} \in K_{\mathfrak{p}}^{\times 2}$. This shows that $\mathfrak{q} \smile \mathfrak{p}$.

For the points whose classes are 2-divisible in the Picard group of $X$, the relation $\smile$ was defined canonically, by inclusion of certain groups (see [1]). Here, for general points, the relation is defined using a (bit esoteric) element $\lambda_{\mathfrak{p}}$, that depends not only on the point $\mathfrak{p}$ but also on the specified system of parameters $\pi_{1}, \ldots, \pi_{k}$. While the dependence on the system of parameters is unavoidable (see the remark below), we may still replace a single square class $\lambda_{\mathfrak{p}}$ by a certain subgroup of $K^{\times} / K^{\times 2}$. Let $\mathfrak{p} \in Z$ be a point, write $\Lambda_{\mathfrak{p}}$ for the subgroup of $\mathbf{E}_{Z \backslash\{\mathfrak{p}\}} / \boldsymbol{\Delta}_{X \backslash\{\mathfrak{p}\}}$ generated by the class of $\lambda_{\mathfrak{p}}$. If $[\mathfrak{p}] \in 2$ Pic $X$, then it follows from Proposition 4 and Proposition 5 that $\Lambda_{\mathfrak{p}}=\mathbf{E}_{X \backslash\{\mathfrak{p}\}}=\mathbf{E}_{Z \backslash\{\mathfrak{p}\}}$. On the other hand, if $[\mathfrak{p}] \notin 2 \mathrm{Pic} X$, then Proposition 8 asserts that

$$
\Lambda_{\mathfrak{p}}=\Delta_{X \backslash\{\mathfrak{p}\}} \dot{\cup} \lambda_{\mathfrak{p}} \Delta_{X \backslash\{\mathfrak{p}\}}
$$

Lemma 6 yields:

ObServation 10. Let $\mathfrak{p}, \mathfrak{q} \in Z$ be two square classes congruent modulo 2 Pic $X$, then $\mathfrak{p} \smile \mathfrak{q}$ if and only if $\Lambda_{\mathfrak{p}}$ is contained in $K_{\mathfrak{q}}^{\times 2}$.

REMARK. As in the previous remarks, let $K=\mathbb{F}_{q}(t), \mathfrak{b}$ be the point at infinity, $\pi=1 / t$ and $\zeta \in \mathbb{F}_{q}$ be a fixed non-square. Take two polynomials of degree 1: $f=t-a, g=t-b$ and set $\mathfrak{p}:=\langle f\rangle, \mathfrak{q}:=\langle g\rangle$. Then $\mathfrak{p}$ is related to $\mathfrak{q}$ with respect to $\pi, \mathfrak{b}, \zeta$ if and only if $(b-a) \in \mathbb{F}_{q}^{\times 2}$. Now perform a change of variables. Set $x:=\zeta t$ and $\pi^{\prime}:=1 / x$. Then $\pi^{\prime}, \mathfrak{b}, \zeta$ also satisfy AS1 - AS4. The field has not changed, we have $K(x)=K(t)$. The primes $\mathfrak{p}, \mathfrak{q}$ stay intact. But the change of the parameter alters the square classes $\lambda_{\mathfrak{p}}, \lambda_{\mathfrak{q}}$. Now, we have $\lambda_{\mathfrak{p}}^{\prime}=\zeta f$ and $\lambda_{\mathfrak{q}}^{\prime}=\zeta g$. Consequently $\mathfrak{p}$ is related to $\mathfrak{q}$ with respect to $\pi^{\prime}, \mathfrak{b}, \zeta$ if and only $\zeta \cdot(b-a) \in \mathbb{F}_{q}^{\times 2}$ if and only if $b-a \notin \mathbb{F}_{q}^{\times 2}$. This shows that the dependence on the selected system of parameters is inherent to the very nature of the relation $\smile$. It can be avoided only for points whose classes sit in 2 Pic $X$, since such classes have zero coordinates with respect to every basis of Pic $X / 2 \operatorname{Pic} X$.

The set $Z$ equipped with the relation $\smile$ (with respect to some fixed system of parameters) forms an undirected graph. Denote this graph by $\mathscr{Z}$. First we prove that $\mathscr{Z}$ does not contain a vertex adjacent to all other vertices. The next proposition generalizes [1. Proposition 15].

Proposition 11. For every point $\mathfrak{p} \in Z$ there is a point $\mathfrak{q} \in Z$ congruent to $\mathfrak{p}$ modulo 2 Pic $X$ and such that $\mathfrak{p} \nsucc \mathfrak{q}$.

Proof. Let $\varepsilon_{1}, \ldots, \varepsilon_{k}$ be the coordinates of $[\mathfrak{p}]+2 \operatorname{Pic} X$ with respect to the basis $\left\{\left[\mathfrak{b}_{i}\right]+\right.$ $2 \operatorname{Pic} X \mid i \leq k\}$. Fix an element $\mu \in K^{\times}$such that $\operatorname{ord}_{\mathfrak{p}} \mu=0$ and $\mu \notin K^{\times 2}$. Then [4, Lemma 2.1] asserts that there is $\lambda \in K^{\times}$and $\mathfrak{q} \in Z \backslash\{\mathfrak{p}\}$ satisfying: 
- $\operatorname{ord}_{\mathfrak{q}} \lambda=1$,

- $\operatorname{ord}_{\mathfrak{p}}(\lambda-\mu) \geq 1$,

- $\operatorname{ord}_{\mathfrak{b}_{i}}(\lambda-1) \geq 1$ for $i \leq k$ if $\varepsilon_{i}=0$,

- $\operatorname{ord}_{\mathfrak{b}_{i}}\left(\lambda-\pi_{i}\right) \geq 1$ for $i \leq k$ if $\varepsilon_{i}=1$,

- $\operatorname{ord}_{\mathfrak{r}} \lambda \equiv 0(\bmod 2)$ for every $\mathfrak{r} \in Z \backslash\{\mathfrak{q}\}$.

It follows that the divisor of $\lambda$ has a form

$$
\operatorname{div}_{X} \lambda=\mathfrak{q}+\sum_{i \leq k} \varepsilon_{i} \mathfrak{b}_{i}+2 \mathcal{D}
$$

for some $\mathcal{D} \in \operatorname{Div} X$. This means that $[\mathfrak{q}] \equiv[\mathfrak{p}](\bmod 2 \operatorname{Pic} X)$. Moreover $\lambda \cdot K^{\times 2}=\lambda_{\mathfrak{q}}$ and since $\lambda$ is congruent to $\mu$ modulo $K_{\mathfrak{p}}^{\times 2}$ we obtain $\mathfrak{q} \nsucc \mathfrak{p}$.

Corollary 12. The graph $\mathscr{Z}$ is not complete.

Finally we compute the diameter of $\mathscr{Z}$. The following theorem is analogous to a similar result for points whose classes are 2-divisible in Pic $X$ (see [1, Theorem 16]), but the proof presented here uses different techniques.

ThEOREM 13. The graph $\mathscr{Z}$ is connected and its diameter equals 2.

Proof. It follows from the previous proposition that the diameter of $\mathscr{Z}$ cannot be equal to 1 . Thus we must show that for every two points $\mathfrak{p}_{1}, \mathfrak{p}_{2} \in Z$, there is another point $\mathfrak{q}$ such that $\mathfrak{p}_{1} \smile \mathfrak{q} \smile \mathfrak{p}_{2}$. To this end, as in the proof of Proposition 11, we use [4, Lemma 2.1]. It asserts that there is $\lambda \in K^{\times}$and $\mathfrak{q} \in Z \backslash\left\{\mathfrak{p}_{1}, \mathfrak{p}_{2}\right\}$ such that

(1) $\operatorname{ord}_{\mathfrak{q}} \lambda=1$,

(2) $\operatorname{ord}_{\mathfrak{p}_{1}}\left(\lambda-\lambda_{\mathfrak{p}_{1}}\right) \geq 1, \operatorname{ord}_{\mathfrak{p}_{2}}\left(\lambda-\lambda_{\mathfrak{p}_{2}}\right) \geq 1$,

(3) $\operatorname{ord}_{\mathfrak{b}}(\lambda-1) \geq 1$ for every $\mathfrak{b} \in \mathfrak{B}$,

(4) $\operatorname{ord}_{\mathfrak{r}} \lambda \equiv 0(\bmod 2)$ for every $\mathfrak{r} \in Z \backslash\left\{\mathfrak{p}_{1}, \mathfrak{p}_{2}, \mathfrak{q}\right\}$.

Therefore $\mathfrak{q}$ is the unique point of $X$, where $\lambda$ has an odd valuation. It follows from [2, Proposition 3.2] that $[\mathfrak{q}] \in 2 \operatorname{Pic} X$. Thus the coordinates of $[\mathfrak{q}]+2$ Pic $X$ with respect to the basis $\{[\mathfrak{b}]+2$ Pic $X \mid \mathfrak{b} \in \mathfrak{B}\}$ are all zero. Consequently points (1) and (3) imply that $\lambda \cdot K^{\times 2}=\lambda_{\mathfrak{q}}$. It follows from (2) that $\lambda$ is a local square simultaneously at $\mathfrak{p}_{1}$ and $\mathfrak{p}_{2}$. Hence $\mathfrak{q} \smile \mathfrak{p}_{1}$ and $\mathfrak{q} \smile \mathfrak{p}_{2}$.

\section{References}

[1] A. Czogała, P. Koprowski, Graph of even points on an arithmetic curve, arxiv:1904.12177.

[2] A. Czogała, P. Koprowski, B. Rothkegel, Wild and even points in global function fields, Colloq. Math. 154 (2018), 275-294.

[3] A. Czogała, P. Koprowski, B. Rothkegel, Wild sets in global function fields, Math. Slovaca 70 (2020), 259-272.

[4] D. B. Leep, A. R. Wadsworth, The Hasse norm theorem mod squares, J. Number Theory 42 (1992), 337-348. 
